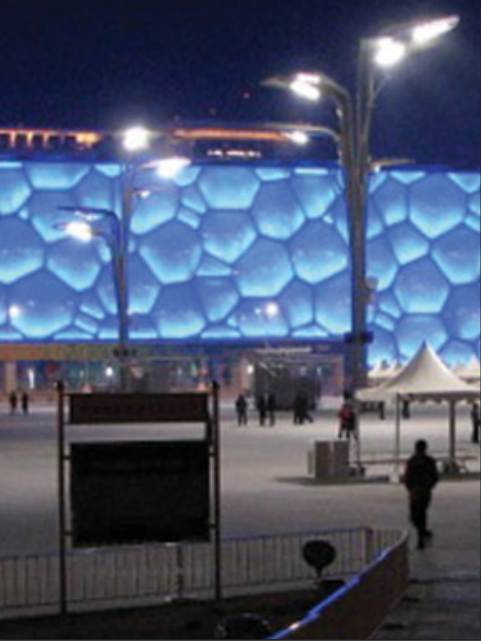

\title{
Solid-state lighting: The future looks bright
}

\author{
Prachi Patel
}

LEDs are gaining traction in lighting, but several research advances are needed before they find widespread use in homes and offices. With increasing funding from government and industry, this could happen within 10 years.

$\mathbf{T}$ he phaseout of the incandescent light bulb in 2005 in Brazil and Venezuela marked the beginning of the end of a 130year old technology. Many countries have since followed suit, and it is now time to move on to something better.

Compact fluorescent lamps (CFLs) are the current chosen alternative. They use $75 \%$ less power and last 10 times longer. Plus, they can produce five times as much light perceived by the human eye for the same amount of power - a key measure called efficacy with units of lumens/Watt. However, CFLs contain toxic mercury, and their electricity-to-light conversion efficiency is unlikely to exceed $20 \%$.

Solid-state lighting is a more promising heir to light bulbs. The best white light-emitting diodes (LEDs) on the market today are $30 \%$ efficient, with efficacies of $130 \mathrm{~lm} / \mathrm{W}$ compared to a CFL's 35-80 lm/W. LEDs last three times as long as CFLs, use non-toxic semiconductor materials, and achieve full brightness immediately. The technology also can closely mimic natural sunlight, which incandescent bulbs achieve by emitting hues covering the entire visible spectrum.

Perhaps most enticing is an LED's potential to reach efficiencies around $70 \%$, corresponding to $286 \mathrm{~lm} / \mathrm{W}$ efficacy. That could make a big dent in the world's energy consumption, provided people do not use more of the high-efficiency light. Lighting uses nearly a fifth of the world's electricity, most of it for commercial, public, and residential buildings, which leads to 1900 megatons of carbon emissions per year, according to the International Energy Agency. High-efficiency LED light sources could slash this consumption by half.

Since LEDs are made from semiconductors, the materials behind digital electronics, they offer the unique capability to be integrated with sophisticated sensors and controls. Innovative LED smart lighting would boost energy savings and make people more productive by adapting to a room's layout or by adjusting brightness to the season or time of day. Significant research hurdles remain, but the solid-state lighting community is confident that extremely efficient, high-quality, affordable white LED light sources should be available within the next 10 years.

"Recent market forecasts suggest that as much as half or more of new lighting installations could be solid state in a few years," said Fred Welsh, who coordinates the U.S. Department of Energy's solid-state lighting Roadmap. "[LED lights] will never be as cheap as incandescent bulbs, but they last a long time, save a lot of energy, and will be cost-effective. People are willing to pay a premium as long as the product performs."

Inorganic LEDs have come a long way since Nick Holonyak made the first dim red device in 1962. In that same decade, Isamu Akasaki started his research on blue LEDs at Nagoya University in Japan, and, building on Akasaki's materials groundwork, Shuji Nakamura at Nichia Corporation demonstrated the first high-brightness blue LED in 1993. By the late 1990s, LEDs were in traffic lights and outdoor displays.

Today, the use of white LEDs in cellphone and TV backlighting is soaring. This has benefited the lighting industry, which "is only seven percent of the market for high-brightness LEDs," said Welsh. White LEDs are making headway in street, parking, and architectural lighting, where the higher cost is offset by low maintenance costs and long lifetimes. The city of Los Angeles is in the midst of retrofitting 140,000 street lamps with LED units, which will cut energy use by $40 \%$ and carbon emissions by 40,500 tons, while saving the city $\$ 10$ million annually.

Gaining a foothold in indoor lighting, however, will require reducing the $\$ /$ lumen cost of high-quality white light. Prices of \$13 per kilolumen for bluish cool white LEDs and \$18 per kilolumen for the yellower warm white LEDs (\$50 for a LED lamp that includes a power supply) in 2010 were still too expensive for home and office buyers. The much-preferred warm white LEDs are also less efficient.

The DOE's Roadmap is targeting a cost of $\$ 1$ per kilolumen for 2020, at efficiencies of $48 \%$ and efficacies over $250 \mathrm{~lm} / \mathrm{W}$ for both cool and warm white LEDs. Past targets have often been surpassed, and experts believe that these will too with continued research advances. For example, advances presented at the recent International Conference on Nitride Semiconductors in Glasgow, Scotland in mid-July showed that researchers are already pushing the materials and research frontiers underlying solid-state lighting.

LEDs are devices in which a 2 nm-thick semiconductor layer, such as indium gallium nitride ( $\mathrm{InGaN})$, is sandwiched between two thicker layers of gallium nitride $(\mathrm{GaN})$, one rich 
in electrons and the other in positively charged particles called holes. A voltage applied across the sandwich injects electrons and holes into the central InGaN layer, where they recombine and emit single-color light depending on the material and layer thicknesses. White LEDs typically use blue GaN chips with a phosphor that converts some of the blue light into yellow. Red phosphors are added to make the light warmer.

The biggest issue with GaN devices is that at the higher currents required for general lighting, the efficiency plummets. The mechanism behind this droop is not fully understood, and it is an issue of active research and intense debate. Several experimental studies have assigned the efficiency loss to Auger recombination, an effect in which an electron and a hole can recombine non-radiatively, transferring energy to a third charge carrier instead of producing a photon. Because three charge carriers are involved, the effect should manifest itself at higher current densities. The precise form of Auger recombination responsible for droop is under debate, while researchers have also proposed alternative theories, claiming that the Auger effect should be small.

"People have tried to overcome [droop] by using more LEDs in a lamp," Welsh said. "It may take a new material system to solve the problem."

The phosphor approach has inherent issues. The conversion of blue wavelengths to longer yellow and red wavelengths entails an unavoidable 20-25\% energy loss. Red phosphors also emit part of their light outside the visible spectrum, which reduces efficiency. Phosphors with narrow emission spectra

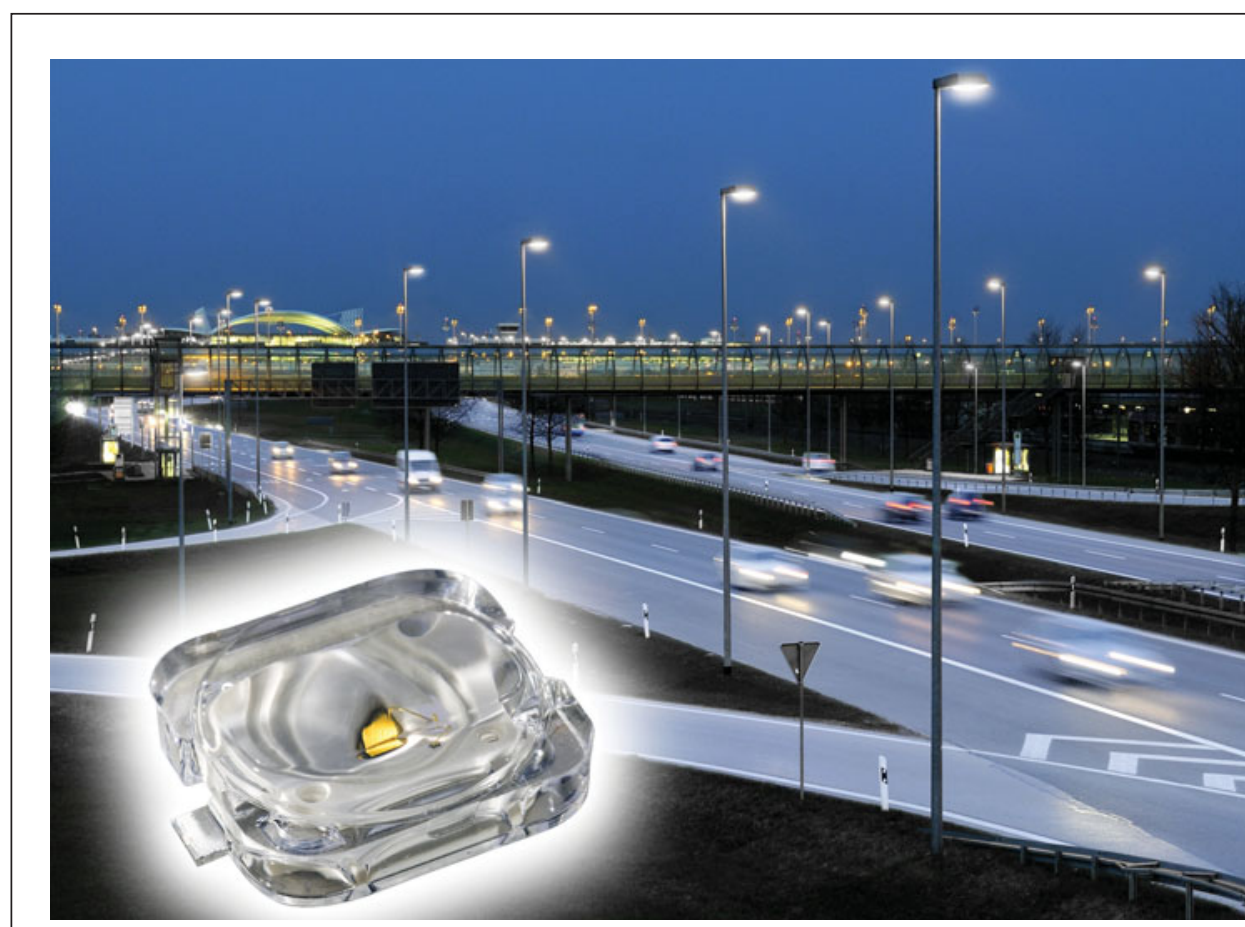

Lighting up roads with LEDs could save energy and cut maintenance costs while considerably reducing light pollution, since light is directed only on the area to be illuminated and not upward.

Credit: OSRAM Opto Semiconductors. could solve this problem. Some manufacturers have used a different method: they add a red light-emitting semiconductor layer to their blue LED chips.

Mixing red, green, blue, and amber LEDs would be the best way to make high-quality white light with efficacy close to 270 $\mathrm{lm} / \mathrm{W}$. This approach has not been popular because red, green, and amber devices are less efficient than their blue counterparts. In the lab at low currents, red, green, and amber LEDs show efficiencies of $75 \%, 40 \%$, and $20 \%$, respectively, compared to $80 \%$ for blue. Efficiencies on that order for each color would be required to make the color-mixing approach feasible.

While fundamental materials advances could improve the efficiency and quality of white LEDs, cost reductions will come from the manufacturing side. For instance, growing GaN LEDs on larger sapphire substrates should produce a higher yield. Researchers at Cambridge University are looking at growing devices on cheaper silicon substrates, for which fabrication equipment has been honed in the electronics industry. Until a few years ago, companies commonly used recycled silicon fabrication machines for LED materials, but they are now starting to use LED-specific machines. "These new machines do things better and faster," said Welsh. "The consistency and quality of products coming out is key."

Even as manufacturers refine LED products to replace traditional light bulbs, engineers are exploring next-generation lighting systems that defy convention. "The idea is to leverage the digital capability of LEDs with advanced sensors and controls to maximize energy savings," said Bob Karlicek, the director of the Smart Lighting Engineering Research Center at the Rennselaer Polytechnic Institute in Troy, NY.

Lights connected to motion sensors and dimmers use $40 \%$ less energy than those without, Karlicek said, but LED smart lighting being developed at the ERC could give even larger energy savings. Switch-free lights would sense their environment and adapt to it by communicating with each other and shining light where and when it is needed. The lights communicate by imperceptibly blinking at high frequencies.

"You can create a baseline optical fingerprint for a room when it is lit with and without sun, morning versus afternoon," Karlicek said. "When you walk into the room, the light flow is altered by your presence. Now you can potentially create a system where you do light sensing and create a notion of where light has to be on 


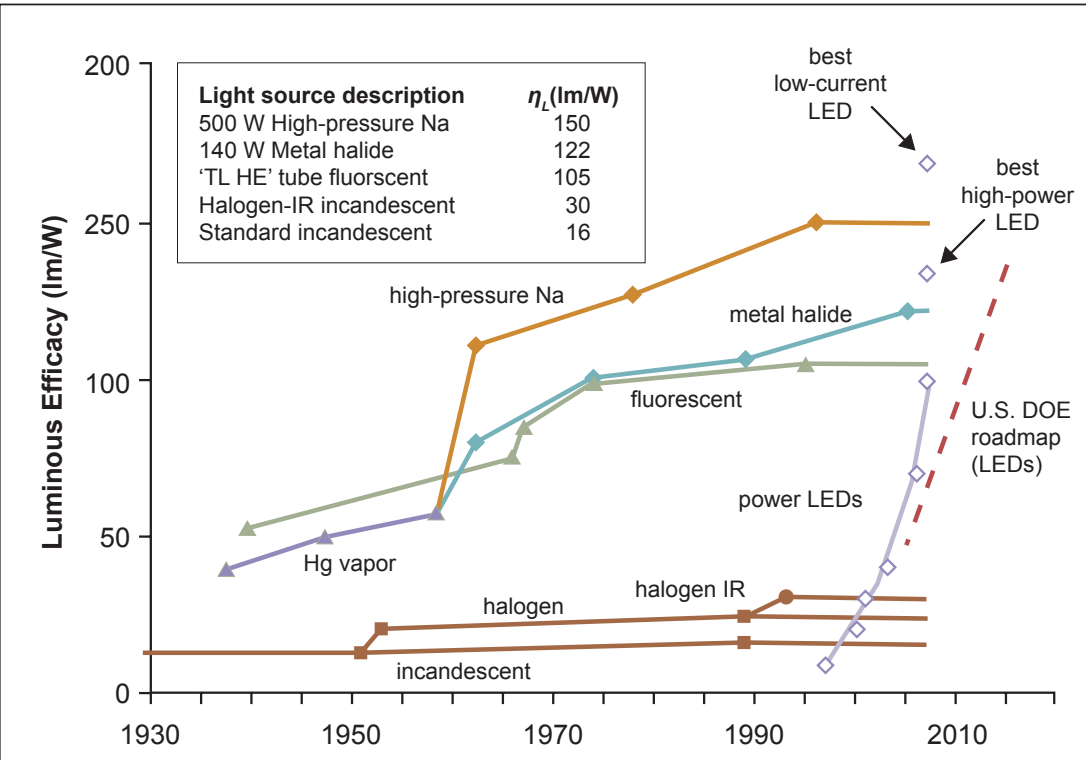

Light-emitting diodes versus conventional light sources. (C) 2007, IEEE
States invested about half that amount in industries and universities. The U.S. government's stimulus program is funding municipalities to install LED lamps on streets and parking lots.

China, though, is leading the LED lighting revolution. The government is offering tax incentives, equipment subsidies, and R\&D funding to companies; funding demonstration projects such as the architectural lighting at the 2008 Olympics; and providing subsidies for LED street, tunnel, and subway lamp installations. Wu Ling, the secretary-general of the China Solid-State Lighting Alliance, said these incentives are driven by the need to develop sustainable economic growth and to save energy. China's lighting consumption increases by $10 \%$ every year, and the implications of switching to LED lighting are tremendous. If the aver- and how intensive depending on layout and people, and the light can adapt automatically."

Government and industry support for LED lighting is growing worldwide. The United Kingdom and France have strong research programs and are backing start-ups. Business sectors in Taiwan and South Korea are aggressively spending on research and development (R\&D). Taiwan's investment in 2010 was over $\$ 600$ million, the most in the world, while the United age LED fixture efficiency reaches $150 \mathrm{~lm} / \mathrm{W}$ and LED lighting takes half the market, the country could save 340 billion $\mathrm{kWh}$ in energy use, about a tenth of its energy consumption in 2009.

The massive initiative in Asia is helping to drive down the cost of solid-state lighting, Karlicek said. "In 10 years, there will be major price reductions in LED lighting, and we'll start to see major impacts."

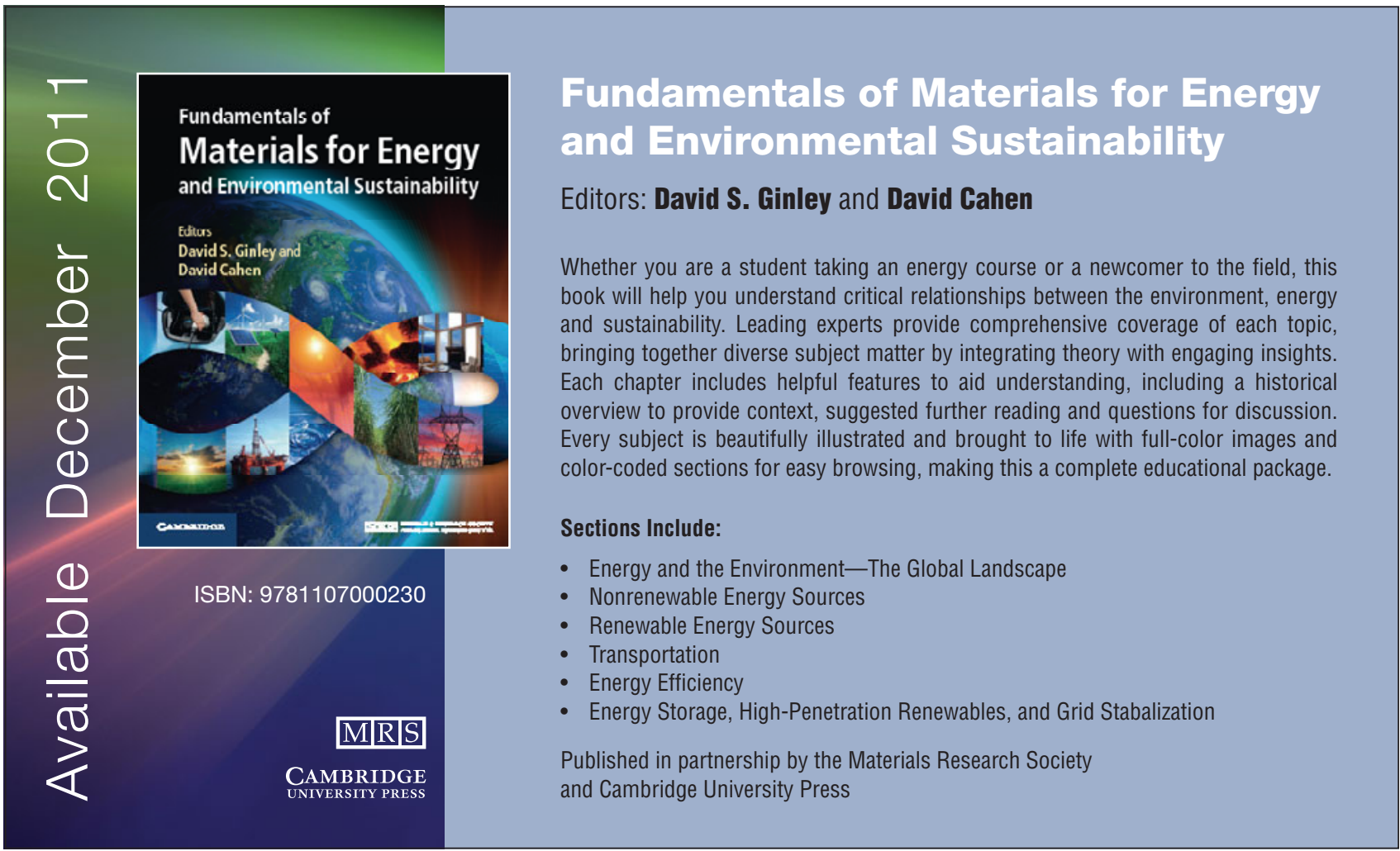

